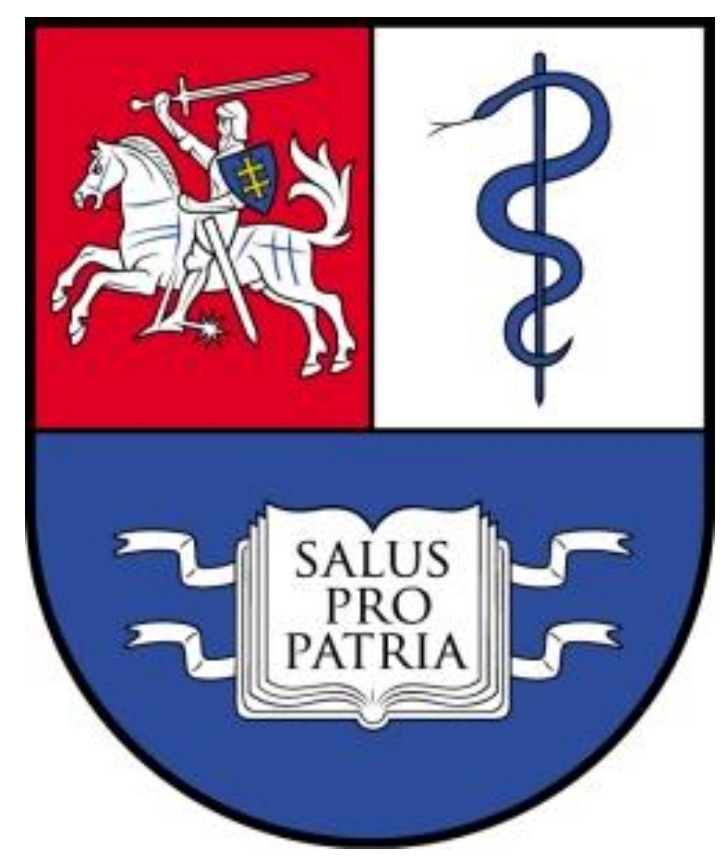

\title{
Time intervals of anaesthesia-induction and decision-to-delivery in different urgency categories for caesarean section
}

\section{Sasnauskaite , K. Rimaitis', V. Baliuliene', A. Macas' \\ 1Department of Anaesthesiology, Medical Academy, Lithuanian University of Health Sciences - Kaunas}

Key words: obstetric anaesthesia, regional anaesthetic techniques, general anaesthesia.

Background and Goal of Study: A first choice of anaesthesia for urgency categories (UC) 1 to 3 caesarean sections (CS) is regional anaesthesia (RA). However, general anaesthesia (GA) is sometimes performed expecting shorter delivery time. The goal of our study was to evaluate time differences between GA and RA in each urgency category of CS.

Materials and Methods: A prospective study was carried out in LUHS, Dept. of Obstetrics from January to December, 2016. CS were classified using Lucas fourgrade classification system. Anaesthesia-induction time and decision-to-delivery interval were compared between spinal anaesthesia (SA), GA and epidural anaesthesia (EA) in each UC. Statistical analysis was performed using Student's t-test, ANOWA and Kruskall-Wallis tests. P $<0.05$ was considered statistically significant.

Results and Discussion: There were 1677 CS included in our study. UC I - 124 (8.0\%), UC II - 344 (22.3\%), UC III - 431 (25.7\%) and UC IV - $643(41.7 \%)$ patients. SA was performed for $1226(73.2 \%)$, GA - 194 (11.6\%), EA for 254 (15.2\%) patients. The differences between SA, GA, EA anaesthesia-induction time and decision-todelivery interval in different UC are shown in table 1.

Conclusion: GA has the shortest anaesthesia-induction time and EA has the shortest decision-to-delivery interval in UC I-III CS.

Table 1. Time intervals between SA, GA and EA in different UCs. Data are presented as Mean $\pm \mathrm{SD}$, and number of cases are given in brackets.

Figure 1. Distribution of SCs by urgency category.

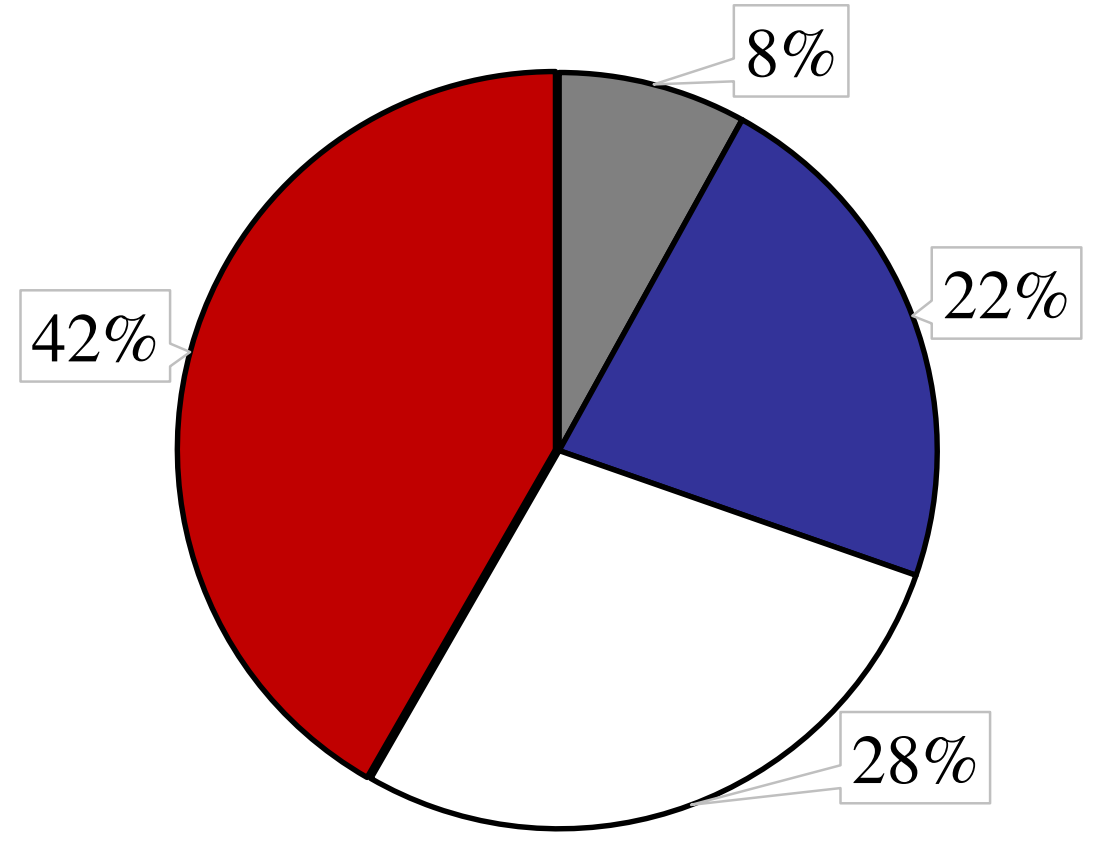

口UC-I $\square$ UC-II $\square$ UC-III $\square$ UC-IV

Figure 2. Anaesthesia techniques selected for SCs.

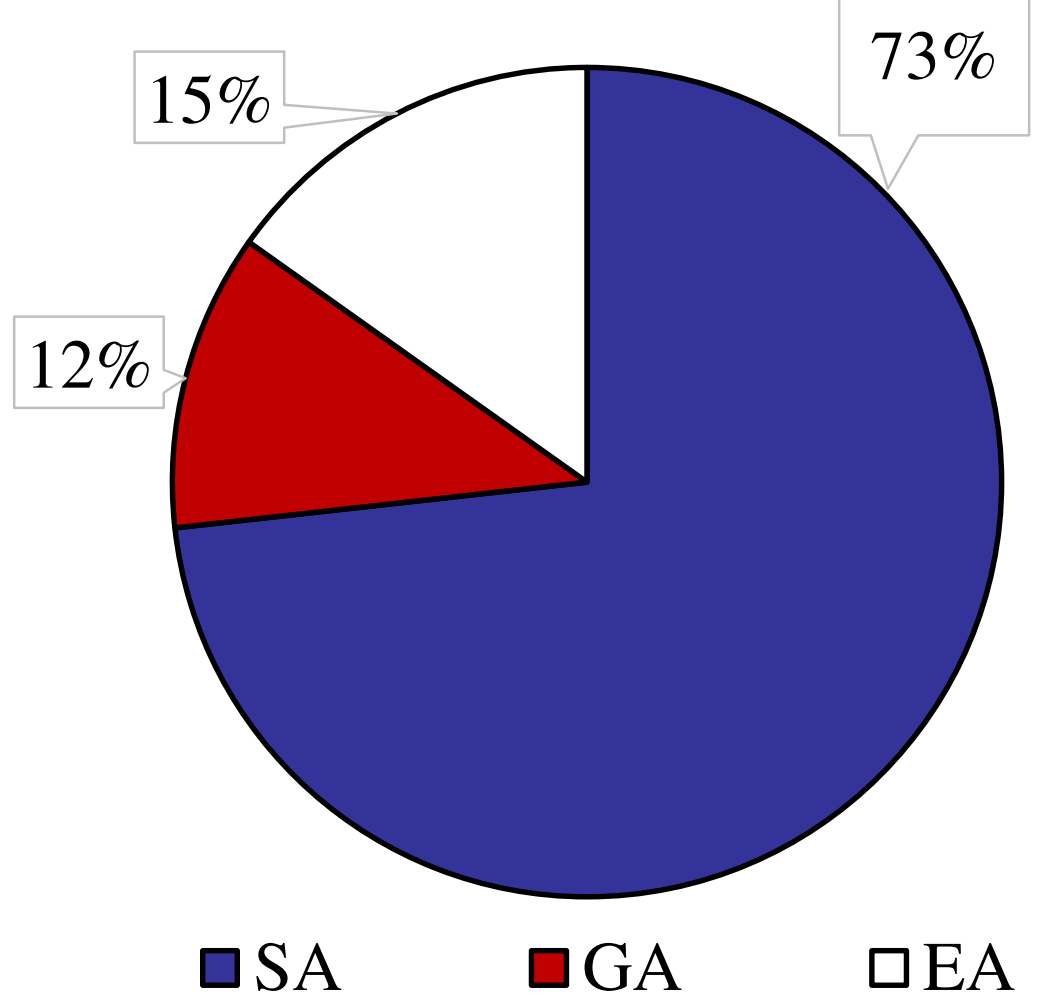

\begin{tabular}{|c|c|c|c|c|c|}
\hline $\begin{array}{l}\text { Urgency } \\
\text { category }\end{array}$ & $\begin{array}{c}\text { Type of } \\
\text { anaesthesia }\end{array}$ & $\begin{array}{l}\text { Anaesthesia- } \\
\text { induction time } \\
\text { (min) }\end{array}$ & P value & $\begin{array}{c}\text { Decision-to- } \\
\text { delivery interval } \\
\text { (min) }\end{array}$ & $\begin{array}{c}\mathrm{P} \\
\text { value }\end{array}$ \\
\hline I & SA & $6.14 \pm 1.10(34)$ & \multirow{3}{*}{0.001} & $46.76 \pm 19.73(34)$ & \multirow{3}{*}{0.001} \\
\hline $\mathbf{I}$ & GA & $2.07 \pm 1.85(67)$ & & $25.46 \pm 13.34(67)$ & \\
\hline I & EA & $13.86 \pm 3.32(22)$ & & $27.68 \pm 8.13(22)$ & \\
\hline II & SA & $6.82 \pm 2.13(190)$ & \multirow{3}{*}{0.001} & $59.90 \pm 46.67(177)$ & \multirow{3}{*}{0.001} \\
\hline II & GA & $4.02 \pm 4.21(34)$ & & $69.39 \pm 92.97(33)$ & \\
\hline II & EA & $13.21 \pm 4.04(120)$ & & $37.31 \pm 19.03(120)$ & \\
\hline III & SA & $7.18 \pm 2.45(327)$ & \multirow{3}{*}{0.001} & $74.27 \pm 52.85(268)$ & \multirow{3}{*}{0.001} \\
\hline III & GA & $2.83 \pm 2.23(36)$ & & $67.72 \pm 48.43(29)$ & \\
\hline III & EA & $13.74 \pm 3.18(66)$ & & $40.96 \pm 23.99$ (66) & \\
\hline IV & SA & $9.48 \pm 4.53(603)$ & \multirow{3}{*}{0.328} & $120.08 \pm 111.03(12)$ & \multirow{3}{*}{0.926} \\
\hline IV & GA & $9.91 \pm 7.51(37)$ & & $131.00(1)$ & \\
\hline IV & EA & $13.33 \pm 2.88$ & & (0) & \\
\hline
\end{tabular}

